age, latin ethnicity and sedentary lifestyle were the RF related to the BMD of the whole body without head (BMDwbwh)

In lumbar BMD, these $4 \mathrm{FR}$ explained up to $85 \%$ of the BMD variation, where the age adds 0'032 per year gained, the male sex subtracts 0'061, the hypovitaminosis D sum 0'077 and the latin ethnicity subtracts 0 '070

Up to a 90 ' $8 \%$ variation of BMDwbwh is explained by these $3 \mathrm{RF}$ : age adds 0'036 per year gained, sedentary life subtracts 0'084 and latin ethnicity subtracts 0'055

Conclusion: The child population at risk of LBM/IOp associates 2 or more risk factors

$8.7 \%$ of children with risk factors have LBM and $4.8 \%$ IOP

The RFs related to changes in BMD are: age, sex, sedentary lifestyle and ethnicity. Hypovitaminosis D correlated positively with lumbar BMD

Disclosure of Interests: None declared

DOI: 10.1136/annrheumdis-2019-eular.3451

\section{AB1014 USE OF ETANERCEPT BIOSIMILAR IN JIA: PRELIMINARY EXPERIENCE USING REAL WORLD DATA}

Marco Marinelli ${ }^{1}$, Achille Marino ${ }^{1}$, Gabriele Simonini ${ }^{1}$, Rolando Cimaz ${ }^{1}$, Teresa Giani ${ }^{1,2}$. 1 Meyer Children's University Hospital of Florence, Florence, Italy; ${ }^{2}$ University of Siena, Siena, Italy

Background: Biosimilars are biological medical drugs that are almost an identical copy of an original drug, manufactured by a different company. Since last year regional regulations have imposed a non-medical switch from reference etanercept (ETN) originator to biosimilar (SB4). We compared treatment survival on SB4 to reference etanercept, assessing efficacy and safety data in our cohort of patients with JIA.

Objectives: To review clinical charts of JIA patients, including efficacy and safety of ETN biosimilars after transition from originator. Compliance was also assessed.

Methods: This was a retrospective observational study of patients with JIA who switched from reference etanercept to SB4 starting during 2018 in our Pediatric Reumatology Department. Clinical and demographic data were collected from charts and inserted into a dedicated database. relevant data included: age at disease onset, age at first administration and duration of treatment, if patients had remission of disease with etanercept therapy or if there were any relapses, ESR and CRP values before and after etanercept therapy, ESR and CRP values before switch to SB4 and after SB4 onset, any adverse effects or relapses of disease after biosimilar.

Results: A total of 14 patients $(13 \mathrm{~F}, 1 \mathrm{M})$ were identified. Age at diagnosis ranged from 1 to 12 years. Before ETN, all had received methotrexate. ETN was added for disease activity persistence, and induced remission in all cases but one. SB4 treatment duration ranged from 1 to 11 months. After switch to SB4 no disease recurrence was observed CRP levels, initially elevated in $8 / 14$ cases, normalized during reference etanercept treatment and remained within normal values in all cases during SB4 treatment. ESR median value initially was $40 \mathrm{~mm} / \mathrm{h}$ (elevated), normalized during reference etanercept treatment (ESR median value 7 $\mathrm{mm} / \mathrm{h}$ ) and remained within normal values (ESR median value $8 \mathrm{~mm} / \mathrm{h}$ ) in all cases during SB4 except one. No side effects were seen, and all families accepted willingly the new prescription.

Conclusion: Our preliminary experience shows that a switch from originator to a biosimilar did not lead to loss of efficacy or new safety signals. Our preliminary results suggest that transitioning from reference etanercept to SB4 is associated with sustained efficacy and no change in the adverse event profile. In conclusion SB4 may provide therapeutically equivalent alternative in pediatric patients with JIA.

Disclosure of Interests: None declared

DOI: 10.1136/annrheumdis-2019-eular.4597

\section{AB1015 CENTRAL NERVOUS SYSTEM VASCULITIS PRECEDING}

A HENOCH-SCHÖNLEIN PURPURA:. ' $C A S E$ REPORT OF A UNCOMMON ASSOCIATION edoardo marrani; ${ }^{2}$ Gabriele Simonini; ${ }^{1}$ Eleonora Fusco; ${ }^{1}$ llaria Maccora; ${ }^{2}$ Anna Rosati; ${ }^{2}$ Rolando Cimaz, Teresa Giani ${ }^{3}$

${ }^{1}$ Postgraduate School of Paediatrics, University of Florence, Florence, Italy, ${ }^{2}$ Anna Meyer Children's Hospital, Florence, Italy, ${ }^{3}$ University of Siena, Department of Medical Biotechnology, Siena, Italy

Background: Henoch-Schönlein purpura (HSP) is a systemic small vessel vasculitis and represent the most common cause of non-thrombocytopenic purpura in children; vasculitis very rarely can involve internal organs. Central nervous system (CNS) is exceptional.

Objectives: We report the case of a patient with CNS vasculitis who presented HSP 4 months after the stroke and during corticosteroid withdrawal.

Methods: Case report

Results: A 9-year-old Caucasian boy was admitted to our hospital due to sudden onset of severe headache and left faciobrachial hemiparesis associated with facial palsy and dysarthria. Brain MRI revealed hyperintense signal changes in amygdala, putamen, and internal capsule, suggestive of recent cerebral ischemic lesion. Magnetic resonance angiography showed asymmetry of internal carotid arteries and middle cerebral artery stenosis, suggestive for a vasculitic-inflammatory nature. He received high dose intravenous methylprednisolone, followed by oral corticosteroids with nearly complete recovery. He was discharged on oral prednisone and low-dose aspirin. In the following months a slow tapering of prednisone was attempted. Four months after the stroke, while receiving prednisolone 0.5 $\mathrm{mg} / \mathrm{kg} / \mathrm{day}$, he presented with sudden onset of purpuric rash on extenso surfaces of the lower extremities, buttocks and arthralgia of the upper and lower extremities. Painful scrotal swelling and abdominal pain were also present. Serial urinalyses were normal. The patient underwent a second MRI of the brain, which ruled out new-onset inflammatory lesions. Therefore, he received intravenous high dose steroids, with complete remission. Sequencing of adenosine deaminase 2 (ADA2) gene ruled out ADA2 deficiency.

Conclusion: We report a case of CNS vasculitis preceding HSP by several months; intriguingly, HSP developed while the patient was under corticosteroid treatment. The exact nature of the association needs to be clarified, to determine if the association was determined by a common pathogenic mechanism or by chance.

\section{REFERENCES}

[1] Akçaboy M, Fidan K, Kandur $\mathrm{Y}$, et al. Cerebral Vasculitis in HenochSchönlein Purpura: A Case Report. Arch Rheumatol. 2017. Mar 24;32 (3):264-267.

[2] Bérubé MD, Blais N, Lanthier S. Neurologic manifestations of HenochSchönlein purpura. Handb Clin Neurol. 2014;120:1101-11.

[3] Suh JS, Hahn WH, Cho BS, Kim SD, Hong IK. A rare case of cerebral vasculitis in Henoch-Schönlein purpura with emphasis on the diagnostic value of magnetic resonance angiography (MRA) and single-photon emission computed tomography (SPECT) given normal magnetic resonance imaging (MRI). Int J Dermatol. 2010 Jul;49(7):803-5.

Acknowledgement: Federica Barbati, MD; Sara Abu-Rumeileh, MD Disclosure of Interests: edoardo marrani: None declared, Gabriele Simonini Grant/research support from: Abbvie, Speakers bureau: Abbvie, Eleonora Fusco: None declared, Ilaria Maccora: None declared, Anna Rosati: None declared, Rolando Cimaz: None declared, Teresa Giani: None declared

DOI: 10.1136/annrheumdis-2019-eular.4663

\section{AB1016 OUTCOME OF TRANSITION OF CARE IN YOUNG ADULTS WITH JUVENILE ONSET CHRONIC RHEUMATIC DISEASES}

Patrícia Martins $^{1,2}$, Sofia C. Barreira ${ }^{1,2}$, Ana Teresa Melo ${ }^{1}$, Raquel CampanilhoMarques $^{1,2}$, Joao Eurico Fonseca ${ }^{1,2}$, Filipa Oliveira-Ramos ${ }^{1,2}$. ${ }^{1}$ Serviço de Reumatologia e Doenças Ósseas Metabólicas, Hospital de Santa Maria, CHULN, Centro Hospitalar Universitário Lisboa Norte, Portugal, Lisbon, Portugal; ${ }^{2}$ Unidade de Investigação em Reumatologia, Instituto de Medicina Molecular, Faculdade de Medicina, Universidade de Lisboa, Centro Académico de Medicina de Lisboa, Portugal, Lisbon, Portugal

Background: The transition process of adolescent care from a paediatric to an adult medical environment may affect the compliance with the management plan. Paediatric care is family oriented and relies on significant parental involvement in decision making. On the contrary, adult care is patient-specific and requires autonomy and independent skills.

Objectives: The aim of this study was to evaluate the transition of care at our centre, namely the adherence to clinical appointments, modification of disease activity and patient satisfaction.

Methods: All consecutive patients with juvenile onset of rheumatic chronic diseases followed in a young adult clinic were included. Disease activity was evaluated at the last appointment in the paediatric unit and up to 2 years after transition of care, according to validated scores for each rheumatic disease. Dropout was defined as not attending the clinic for 2 consecutive visits. Global assessment of patient satisfaction with the clinical 
appointments before and after transition of care was evaluated in a scale of 0 to 10. Variables were analysed as means, medians and frequencies as appropriate. Univariate analysis was performed with student t-test and Qui-square.

Results: 126 patients were included. Of these, $77(61.1 \%)$ had juvenile idiopathic arthritis (JIA, see table I for list of diagnosis), 78 (61\%) were female with a mean age of $23.1 \pm 3.2$ years and a mean disease duration of $12.7 \pm 5.3$ years. During the transition of care, 92 patients were treated with conventional disease modifying antirheumatic drugs (73\%) and 35 with biologic therapy (27.8\%). 69 patients (54.7\%) missed at least one clinical appointment with a dropout rate of $9 \%$. This was associated with longer disease duration (15.9 vs 12.3 years, $p=0.024)$. 11 patients $(8.7 \%)$ had worsened clinical activity: 5 patients with polyarticular JIA with arthritis flare (DDAS28 $2.14 \pm 0.83) ; 4$ patients with oligoarticular JIA with new onset uveitis and 2 patients with juvenile systemic lupus erythematosus with a SLEDAI increase from 5 to 16 points. 4 patients abandoned DMARDs. Regarding patient satisfaction questionnaire, paediatric rheumatology appointments had a median evaluation of 9 (7-10), adult rheumatology appointments of $8(5-10)$ and the transition process had an evaluation of $8(5-10)$. The majority of patients reported the longer appointment waiting time as the major negative aspect after transition. Conclusion: In our centre the transition of care had a small percentage of dropping out from the clinic, which was associated with longer disease duration, a slight worsening of disease activity and a $10 \%$ decrease in patient satisfaction.

\section{REFERENCES}

[1] Foster $\mathrm{H}$, et al. EULAR/PReS standards and recommendations for the transitional care of young people with juvenile-onset rheumatic diseases. Annals of the Rheumatic Diseases 2018; 77 960-960 Published Online First: 10 May 2018.

Abstract AB1016 Table 1. Descriptive analyses of diagnosis. SLE - systemic lupus erythematosus. ${ }^{*}$ Mixed connective tissue disease, Systemic sclerosis, Raynaud phenomenon, Overlap syndrome, Osteoporosis, Osteogenesis Imperfecta, Spondyloarthriti

\begin{tabular}{lc}
\hline Diagnosis & $\mathbf{N}(\%)$ \\
\hline JIA & $77(61.1)$ \\
Persistent Oligoarthritis & $22(17.5)$ \\
Enthesitis related arthritis & $22(17.5)$ \\
Rheumatoid factor - polyarthritis & $11(8.7)$ \\
Extended Oligoarthritis & $9(7.1)$ \\
Systemic & $6(4.8)$ \\
Psoriatic Arthritis & $4(3.2)$ \\
Rheumatoid factor + polyarthritis & $3(2.4)$ \\
Systemic lupus erythematosus & $14(11.1)$ \\
Vasculitis & $9(7.1)$ \\
Autoinflammatory syndrome & $5(4)$ \\
Dermatomyositis & $3(2.4)$ \\
Other diagnosis* & $19(15.1)$ \\
\hline
\end{tabular}

Disclosure of Interests: None declared

DOI: 10.1136/annrheumdis-2019-eular.8130

\section{AB1017 WHAT IS THE CURRENT SITUATION OF THE PAEDIATRIC RHEUMATOLOGY TRANSITION CARE UNITS?}

José Luis Martín-Varillas ${ }^{1}$, Daniel Clemente ${ }^{2}$, J.C. López Robledillo ${ }^{2}$, Belén Serrano Benavente ${ }^{3}$, Indalecio Monteagudo ${ }^{3}$, Juan Carlos Nieto ${ }^{3}$. ${ }^{1}$ H.U.M. Valdecilla, Santander, Spain; ${ }^{2}$ H.U. Niño Jesús, Madrid, Spain; ${ }^{3}$ H.G.U. Gregorio Marañón, Madrid, Spain

Background: Switching from paediatric rheumatology to the adult rheumatology care units has a negative impact on disease control and adherence in young patients with juvenile idiopathic arthritis (JIA) but also in other chronic diseases. Transitional care services provide the opportunity of being followed-up by an adult rheumatology specialized in juvenile rheumatic diseases.

Objectives: In our study we report the current situation of a rheumatology transition care unit (RTCU).

Methods: We set up an observational and retrospective study of patients followed in our unit between September 2015 and December 2018. We included those patients with a disease beginning at 16 years or younger and those patients who were attended on RTCU for the first time before age 25. Clinical and demographic data and all treatments received by the patients before and after de first visit in the transition care unit were also collected.
Results: 119 patients were attended in our RTCU. After applying the exclusion criteria, 110 patients remained: 78 female/32 male $(70.9 / 29.1 \%)$ with a median age of 18.5 [17.9-19.8] years at the first visit. No significant differences in age at the first visit were found between the first (September-2015 to September-2017) and the second stage (October2017 to December-2018), $18.7(17.8-20.2)$ vs. 18.2 (17.9-18.6) years respectively. The most frequent diagnoses are included in the table 1 .

Of the 75 patients with JIA, 54 were women (72\%) with a median age at the disease onset of 6.88 [2.3-12.7] and $21.3 \%$ of patients had chronic anterior uveitis. 22 patients $(29.3 \%)$ presented joint or ocular inflammatory activity or flare at the time of transition first visit but only 1 patient maintained inflammatory activity during the 3 years of follow-up. A total of 64 patients $(85.3 \%)$ with JIA diagnosis were under treatment with synthetic DMARD, mostly methotrexate (61) or leflunomide (7). In addition, 43 patients (57.3\%) required biological DMARD in the past, and 8 of them (10.7\%) required more than one. The most frequently used drugs were: etanercept (ETN) (29 patients) and adalimumab (ADA) (19 patients). Tocilizumab (TCZ) (4), abatacept (1) and infliximab (1) were also used.

Of these patients, at the time of the first visit on RTCU, 24 (32\%) had no treatment, 42 patients $(56 \%)$ had synthetic DMARD and 34 patients $(45.3 \%)$ had biologic therapy in monotherapy or combined with DMARDs (19 ETN, 12 ADA, and $3 \mathrm{TCZ}$ ).

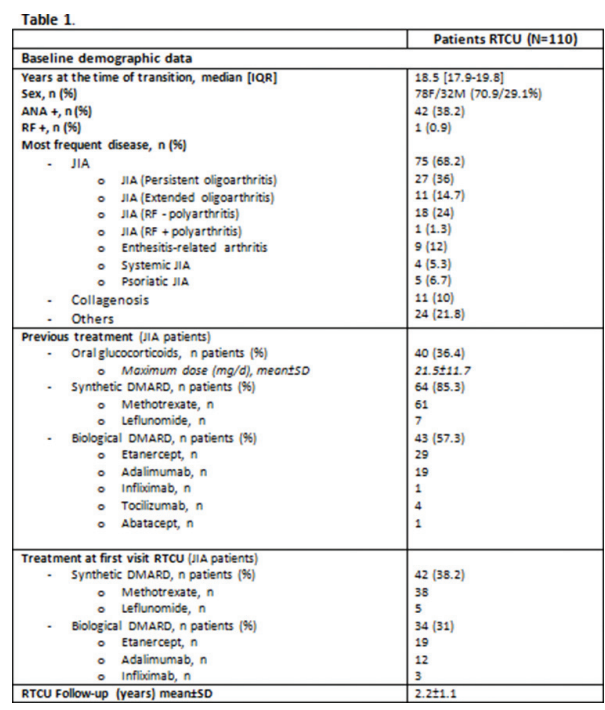

Conclusion: RTCU assessed mainly patients with JIA and connective tissue diseases. Most patients were inactive during the transfer to the adult unit, but the existence of patients that were still active and/or needed treatment with synthetic and/or biological DMARD reaffirms the need for close control in this period.

Disclosure of Interests: None declared

DOI: 10.1136/annrheumdis-2019-eular.6899

\section{AB1018 CONE BEAM CT IN JUVENILE IDIOPATHIC ARTHRITIS: ROLE OF IMAGING DIAGNOSTIC TOOL WITH MINIMAL X-RAY EXPOSURE IN THE FREQUENT, SUBTLE AND INDOLENT TEMPOROMANDIBULAR JOINT INVOLVEMENT}

Maddalena Maschio ${ }^{1}$, Sara Pieropan ${ }^{1}$, Giulia Aiello ${ }^{1}$, Giulia Melotti ${ }^{1}$ Elisa Tadiotto ${ }^{1}$, Erica Giacomelli ${ }^{1}$, Antonio D'agostino ${ }^{2}$, Lorenzo Trevisiol ${ }^{2}$, Giorgio Piacentini ${ }^{1} .{ }^{1}$ Azienda Ospedaliera Universitaria Integrata Verona, Pediatric, Verona, Italy, ${ }^{2}$ Azienda Ospedaliera Universitaria Integrata Verona, MAxillo Facial Surgery, Verona, Italy

Background: The prevalence of the temporomandibular joint (TMJ) involvement in patients affected by juvenile idiopathic arthritis (JIA) ranges from $17 \%$ to $87 \%$ depending on population. TMJ is frequently the first and unique joint involved in the arthritic process. Unfortunately detection of TMJ arthritis in children with JIA is difficult as early signs and symptoms are missing in most patients. Therefore failure to diagnose and treat TMJ arthritis may have severe consequences on masticatory function, like 